\title{
On the relation between conservation and dual consistency for summation-by-parts schemes
}

\author{
Jan Nordström and Fatemeh Ghasemi \\ Journal Article
}

Tweet

N.B.: When citing this work, cite the original article.

Original Publication:

Jan Nordström and Fatemeh Ghasemi, On the relation between conservation and dual consistency for summation-by-parts schemes, Journal of Computational Physics, 2017. 344, pp.437-439.

http://dx.doi.org/10.1016/j.jcp.2017.04.072

Copyright: Elsevier

http://www.elsevier.com/

Postprint available at: Linköping University Electronic Press

http://urn.kb.se/resolve?urn=urn:nbn:se:liu:diva-137545

(c)

1.UU UNWENGES 


\title{
On the relation between conservation and dual consistency for summation-by-parts schemes
}

\author{
Jan Nordström \& Fatemeh Ghasemi \\ Department of Mathematics, Computational Mathematics, Linköping University, \\ SE-58183 Linköping, Sweden \\ jan.nordstrom@liu.se, fatemeh.ghasemi@liu.se
}

\section{Introduction}

We consider initial boundary value problems discretized by summationby-parts (SBP) operators together with the simultaneous approximation term (SAT) technique. In $[1,2,3,4]$, it was shown that dual consistent discretizations on SBP-SAT form, leads to superconvergent linear functionals. For multi-block/element SBP-SAT based schemes [5, 6, 7, 8, 9], conservation is one of the most important issues. In this note, we show that dual consistency and conservation are equivalent concepts for linear conservation laws.

\section{The continuous problem}

Consider the two coupled linear conservation laws

$$
\begin{array}{rlrl}
u_{t}+f_{L}(u)_{x} & =0, & -1 \leq x \leq 0, t>0, \\
v_{t}+f_{R}(v)_{x} & =0, & 0 \leq x \leq 1, t>0, \\
f_{L}(u(0, t)) & =f_{R}(v(0, t)), & x & =0, t>0,
\end{array}
$$

augmented with initial conditions. The $m$ component long solution vectors $u$ and $v$ are smooth and continuous, also across the interface. The subscripts $L, R$ refer to the left and right spatial intervals, respectively. For clarity and ease of presentation, we ignore the boundary conditions at $x= \pm 1$.

The coupled problem (1) can be written more compactly as

$$
\begin{aligned}
w_{t}+F(w)_{x} & =0, & -1 \leq x \leq 1, & t>0, \\
f_{L}(u(0, t)) & =f_{R}(v(0, t)), & x & =0, t>0,
\end{aligned}
$$

where the flux is given by

$$
F(w)=\left\{\begin{array}{lr}
f_{L}(u), & -1 \leq x \leq 0 \\
f_{R}(v), & 0 \leq x \leq 1,
\end{array} \text { and } w=\left\{\begin{array}{rr}
u, & -1 \leq x \leq 0 \\
v, & 0 \leq x \leq 1
\end{array}\right.\right.
$$

For future reference we define the inner product as $(g, h)_{a}^{b}=\int_{a}^{b} g^{T} h d x$. 


\subsection{Conservation}

We multiply the equation (1) with the transpose of a smooth vector function $\varphi(x)$ with compact support in $-1 \leq x \leq 1$. Integration in space yields

$$
\begin{aligned}
\frac{d}{d t}(\varphi, w)_{-1}^{1} & =\left(\varphi_{x}, f_{L}(u)\right)_{-1}^{0}-\left.\varphi^{T} f_{L}(u)\right|_{-1} ^{0}+\left(\varphi_{x}, f_{R}(v)\right)_{0}^{1}-\left.\varphi^{T} f_{R}(v)\right|_{0} ^{1} \\
& =\left(\varphi_{x}, f_{L}(u)\right)_{-1}^{0}+\left(\varphi_{x}, f_{R}(v)\right)_{0}^{1} .
\end{aligned}
$$

The relation (3) show that the problem (2) is a conservation law.

\subsection{The dual problem}

The dual problem (see [2]) associated to (1), (2) is

$$
\begin{aligned}
\theta_{\tau}-F(\theta)_{x} & =0, & -1 \leq x \leq 1, & \tau>0, \\
f_{L}(\phi(0, t)) & =f_{R}(\psi(0, t)), & x & =0, \tau>0,
\end{aligned}
$$

augmented with homogeneous initial conditions. In (4), $\theta=\phi \in[-1 \leq x \leq$ $0]$ and $\theta=\psi \in[0 \leq x \leq 1]$. The boundary conditions at $x= \pm 1$ are ignored, and similary to the primal problem, (4) can be shown to be conservative.

\section{The semi-discrete approximation}

We discretize the derivatives by using difference operators on SBP form,

$$
\left(D_{L} \otimes I\right) \mathbf{f}_{L} \approx\left(f_{L}\right)_{x}, \quad\left(D_{R} \otimes I\right) \mathbf{f}_{R} \approx\left(f_{R}\right)_{x}
$$

where $\otimes$ denotes the Kronecker product [10] and $I$ is the $m \times m$ identity matrix. In (5), $D_{L, R}=P_{L, R}^{-1} Q_{L, R}$ where the matrices $P_{L, R}$ are symmetric positive definite, $Q_{L, R}$ are almost skew-symmetric and satisfy the SBP property $Q_{L, R}+Q_{L, R}^{T}=\operatorname{diag}(-1,0, \ldots, 0,1)$.

The semi-discrete SBP-SAT formulation of (2) for $\mathbf{w}=[\mathbf{u}, \mathbf{v}]^{T}$ is

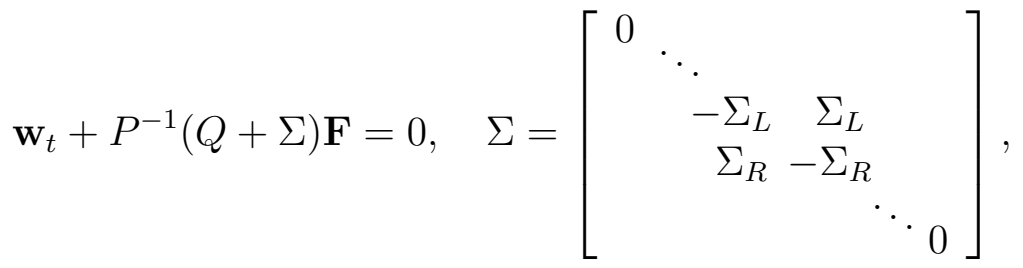

where $\mathbf{F}=\left[\mathbf{f}_{L}, \mathbf{f}_{R}\right]^{T}, Q=\operatorname{diag}\left(Q_{L}, Q_{R}\right) \otimes I$ and $P=\operatorname{diag}\left(P_{L}, P_{R}\right) \otimes I$. 


\subsection{Conservation of the semi-discrete approximation}

Let the discrete scalar product for the two vectors $\mathbf{g}$ and $\mathbf{h}$ be defined by $(\mathbf{g}, \mathbf{h})_{P}=\mathbf{g}^{T} P \mathbf{h}$. Multiplying (6) by $\varphi^{T} P$, with $\varphi=\left[\varphi_{L}, \varphi_{R}\right]^{T}$, where $\varphi$ is a projection of the smooth function $\varphi$ onto the grid. By using the SBP property of $Q_{L}$ and $Q_{R}$, and $\left(\varphi_{L}\right)_{N}=\left(\varphi_{R}\right)_{0}:=\varphi_{I}$ this leads to

$$
\begin{aligned}
\frac{d}{d t}(\boldsymbol{\varphi}, \mathbf{w})_{P}= & -\boldsymbol{\varphi}^{T}(Q+\Sigma) \mathbf{F}=\left(D_{L} \boldsymbol{\varphi}_{L}\right)^{T} P_{L} \mathbf{f}_{L}+\left(D_{R} \boldsymbol{\varphi}_{R}\right)^{T} P_{R} \mathbf{f}_{R} \\
& +\boldsymbol{\varphi}_{I}^{T}\left(\Sigma_{L}-\Sigma_{R}-I_{m}\right)\left[f_{L}\left(\mathbf{u}_{N}\right)-f_{R}\left(\mathbf{v}_{0}\right)\right],
\end{aligned}
$$

where $\mathbf{u}_{N}$ and $\mathbf{v}_{0}$ represent $u(0, t)$ and $v(0, t)$, respectively. The scheme $(6)$ is conservative (compare with (3)) if

$$
\Sigma_{L}=I_{m}+\Sigma_{R}
$$

\subsection{Dual Consistency}

The discrete problem (6) can be rewritten as

$$
\mathbf{w}_{t}+L \mathbf{F}=0, \quad L=P^{-1}(Q+\Sigma) .
$$

The discrete dual problem for $\boldsymbol{\theta}=[\boldsymbol{\phi}, \boldsymbol{\psi}]^{T}$ corresponding to (8) is (see [2])

$$
\boldsymbol{\theta}_{\tau}+P^{-1}(L)^{T} P \mathbf{F}=0 .
$$

Substituting $L$ into (9), and using the SBP property of $Q_{L}$ and $Q_{R}$, leads to

$$
\boldsymbol{\theta}_{\tau}-P^{-1}(Q-\tilde{\Sigma}) \mathbf{F}=0, \quad \tilde{\Sigma}=\left[\begin{array}{ccccc}
0 & & & & \\
& \ddots & & \\
& I_{m}-\Sigma_{L} & \Sigma_{R} & & \\
& \Sigma_{L} & -I_{m}-\Sigma_{R} & \\
& & & \ddots & 0
\end{array}\right] \text {. }
$$

Note that $P^{-1} Q \mathbf{F}$ approximates $F(\theta)_{x}$ in (4). If $P^{-1} \tilde{\Sigma} \mathbf{F}$ impose the dual interface conditions in (4), then the scheme (10) is dual consistent $[1,2]$.

\section{The relation between conservation and dual consistency}

We are now ready to state the main result.

Proposition 1. The scheme (6) is conservative if and only if it is dual consistent.

Proof. The vector $P^{-1} \tilde{\Sigma} \mathbf{F}$ impose the dual interface conditions, if $I_{m}-\Sigma_{L}=$ $-\Sigma_{R}$, which is equal to the conservation condition (7). By inserting the conservation condition (7) into (10) we find that $P^{-1} \tilde{\Sigma} \mathbf{F}$ impose the dual interface conditions, which implies dual consistency of (10). 


\section{References}

[1] J. E. Hicken, D. W. Zingg, Superconvergent functional estimates from summation-by-parts finite-difference discretizations, SIAM Journal on Scientific Computing 33 (2011) 893-922.

[2] J. Berg, J. Nordström, Superconvergent functional output for timedependent problems using finite differences on summation-by-parts form, Journal of Computational Physics 231 (2012) 6846-6860.

[3] J. E. Hicken, D. W. Zingg, Dual consistency and functional accuracy: a finite-difference perspective, Journal of Computational Physics 256 (2014) 161-182.

[4] J. Berg, J. Nordström, On the impact of boundary conditions on dual consistent finite difference discretizations, Journal of Computational Physics 236 (2013) 41-55.

[5] M. H. Carpenter, J. Nordström, D. Gottlieb, A stable and conservative interface treatment of arbitrary spatial accuracy, Journal of Computational Physics 148 (1999) 341-365.

[6] M. H. Carpenter, J. Nordström, D. Gottleib, Revisiting and extending interface penalties for multidomain summation-by-parts operators, Journal of Scientific Computing 45 (2010) 118-150.

[7] J. Nordström, J. Gong, E. van der Weide, M. Svärd, A stable and conservative high order multi-block method for the compressible NavierStokes equations, Journal of Computational Physics 228 (2009) 90209035 .

[8] G. J. Gassner, A skew-symmetric discontinuous galerkin spectral element discretization and its relation to SBP-SAT finite difference methods, Journal of Scientific Computing 35 (2013) 1233-1253.

[9] D. Funaro, D. Gottlieb, A new method of imposing boundary conditions in pseudospectral approximations of hyperbolic equations, Mathematics of Computation 51 (1988) 599-613.

[10] R. A. Horn, C. R. Johnson, Topics in Matrix Analysis, Cambridge University Press, 1991. 